OPEN ACCESS

Edited by:

Debbie Guest

Royal Veterinary College (RVC),

United Kingdom

Reviewed by:

Andreas Kurtz,

Fraunhofer Society (FHG), Germany

Sridhar Goud,

National Institutes of Health (NIH)

United States

*Correspondence:

Ali G. Turhan

turviv33@gmail.com

Annelise Bennaceur-Griscelli

abenna@hotmail.fr

Specialty section:

This article was submitted to

Translational Medicine,

a section of the journal

Frontiers in Medicine

Received: 21 June 2021

Accepted: 17 September 2021

Published: 13 October 2021

Citation:

Turhan AG, Hwang JW, Chaker D,

Tasteyre A, Latsis T, Griscelli F,

Desterke $C$ and Bennaceur-Griscelli A (2021) iPSC-Derived Organoids as

Therapeutic Models in Regenerative

Medicine and Oncology.

Front. Med. 8:728543.

doi: 10.3389/fmed.2021.728543

\section{iPSC-Derived Organoids as Therapeutic Models in Regenerative Medicine and Oncology}

\author{
Ali G. Turhan ${ }^{1,2,3,4,5 *}$, Jinwook W. Hwang ${ }^{1,2}$, Diana Chaker ${ }^{1,2,4,5}$, Albert Tasteyre ${ }^{1,2,4,5}$, \\ Theodoros Latsis ${ }^{1,2,4}$, Frank Griscelli ${ }^{1,2,4,5,6}$, Christophe Desterke ${ }^{1,2,4}$ and \\ Annelise Bennaceur-Griscelli ${ }^{1,2,3,4,5 *}$
}

${ }^{1}$ INSERM UAVO9 UMR-S 935, Université Paris Saclay, Villejuif, France, ${ }^{2}$ ESTeam Paris Sud, Université Paris Saclay, Villejuif, France, ${ }^{3}$ APHP Paris Saclay, Department of Hematology, Hopital Bicetre and Paul Brousse, Villejuif, France, ${ }^{4}$ INGESTEM National iPSC Infrastructure, Villejuif, France, ${ }^{5}$ CITHERA, Centre for IPSC Therapies, INSERM UMS-45, Genopole, Evry, France, ${ }^{6}$ Université Paris Descartes, Faculté Sorbonne Paris Cité, Faculté des Sciences Pharmaceutiques et Biologiques, Paris, France

Progress made during the last decade in stem cell biology allows currently an unprecedented potential to translate these advances into the clinical applications and to shape the future of regenerative medicine. Organoid technology is amongst these major developments, derived from primary tissues or more recently, from induced pluripotent stem cells (iPSC). The use of iPSC technology offers the possibility of cancer modeling especially in hereditary cancers with germline oncogenic mutations. Similarly, it has the advantage to be amenable to genome editing with introduction of specific oncogenic alterations using CRISPR-mediated gene editing. In the field of regenerative medicine, iPSC-derived organoids hold promise for the generation of future advanced therapeutic medicinal products (ATMP) for organ repair. Finally, it appears that they can be of highly useful experimental tools to determine cell targets of SARS-Cov-2 infections allowing to test anti-Covid drugs. Thus, with the possibilities of genomic editing and the development of new protocols for differentiation toward functional tissues, it is expected that iPSC-derived organoid technology will represent also a therapeutic tool in all areas of medicine.

Keywords: induced pluripotent stem (IPS) cell, organoid, cancer, drug discovery, regenerative medicine

\section{INTRODUCTION}

Organoids are tridimensional assembly of cells, mimicking organ-like features generated in vitro under specific cues (1). Their ability of self-organization in vitro under defined conditions allows their development for several days or weeks depending on the conditions of culture. There are now extensive data showing that these structures can recapitulate some of the features observed in adult organs, opening therefore major perspectives for their use in disease modeling, precision oncology and perhaps in the future as tools of regenerative medicine. This technology has also the potential to replace animal experiments as theoretically any tissue can be generated in vitro.

Currently, organoid-like structures have been successfully generated from several human tissues (1). These include essentially heart, digestive system, liver, brain, lung, and kidney organoids. These structures have been used to generate either healthy or diseased tissues, allowing to compare the behavior of different cell populations contributing to the organoid under in vitro conditions. They 
have provided important clues for the identification of new signaling pathways and novel targets especially in precision oncology. However, they require obviously a biopsy which might be difficult to obtain. The advantage of these organoids generated from cancer tissue biopsy is obviously the possibility of obtaining more precise information with regard to the tumor microenvironment as this is discussed below. On the other hand, primary cancer-derived organoids are not amenable to genome editing or extensive long-term cultures. One approach to circumvent these obstacles and which is currently in development is the use of iPSC technology to manufacture different organoids which is the subject of this review.

\section{IPSC-DERIVED ORGANOIDS}

The pioneering work of S. Yamanaka which led to the revolutionary iPSC technology allows the reprogramming of an adult somatic cell toward an embryonic state similar to embryonic stem cells (ESC). Since their initial description in 2006 in mice (2) and in 2007 in human cells (3), iPSC are increasingly studied in stem cell research and more recently in the therapeutic arena by the possibility of generating differentiated cells for therapeutic purposes (4). More recently, the attention was focused on the possibility of generating from pluripotent stem cells (either iPSC or ESC) organ-like structures called "organoids" initially described from the adult tissue samples (1).

The organoid field has emerged from pioneering work of the group of Hans Clevers which has shown initially the possibility of generating gut organoids from Lgr5+ stem cells (5). These findings have now been reproduced and extended to other tissues and organoids have been obtained from several adult tissues. More recently, IPSC-derived organoids came into the forefront of stem cell research, due to the fact that as compared to adult tissue-derived organoids, they offer the possibility to combine the self-organization potential of iPSCs and the possibility of directing these cells toward potentially to any organ-specific differentiation (6).

Based on the events of human embryonic intestinal development, Spence at al first showed the possibility of generating intestinal organoids using a series of successive growth factor additions allowing endoderm induction, patterning and morphogenesis from human embryonic stem cells (H1, H9) and from four lines of iPSC. Interestingly, the 3D intestinal structures showed functional features of intestinal epithelium such as absorption and exocrine functions (7). Similarly, using self-organizing embryonic stem cells, Nakano et al. (8) have shown the possibility of generation of 3D optic cups.

in vitro models of brain development have also been possible with the advent of iPSC-derived organoids technology. In 3D culture systems, it was possible to generate mini-brains with highly specialized zones and structures such as cortex and radial glial cells and to model human microcephaly (9). The initial methodology has now progressed to the stage where it is possible to generate highly specialized cells such as oligodendrocytes and astrocytes (10) as well as long-term culture procedures of cerebral structures leading to highly specialized advanced brain organoids to study later stages of neural development (11). Finally the possibility of generating separately different parts of human brain has been described (12).

In the field of kidney development and kidney diseases, iPSCderived kidney organoids allowed the possibility of generating highly specialized structures such as distal and proximal tubules as well as glomeruli with podocytes with a transcriptomic features similar to that of human fetal kidneys (13). iPSC-derived kidney organoid technology is of interest not only for gaining pathophysiologic insights but also to determine the effects of drug development in the transplant setting, for instance to evaluate the toxicity of tacrolimus (14).

The complex structure of the liver can also be reproduced using iPSC-derived organoid technology. One of these studies has shown the possibility of obtaining transplantable 3D hepatic buds with functional activities by the combined culture of iPSCderived endoderm directed toward hepatic differentiation along with mesenchymal stem cells and the endothelial HUVEC cell line (15) Hepatic organoids can also be obtained from normal or patient-derived iPSC to model human hepatic diseases $(16,17)$. Although adult liver tissue can be targeted to generate hepatic organoids, iPSC-derived liver organoids can have a potential advantage of their expansion ability, which can be of interest for toxicology purposes allowing to screen large numbers of compounds in the industrial setting (17). ESC and iPSC-derived cardiac structures can be obtained with highly reproducible methods, giving rise to contractile structures including the possibility of morphological compartmentations such as cardiac chambers (18). This technology represents also an important tool for drug screening but also for disease modeling using patient-derived iPSC (19) (Figure 1).

The multicellular nature of the lung can also be recapitulated using IPSC-derived organoids (20) allowing generation of 3D structures containing lung progenitors, alveolar type 2 (AT2) cells as well as airway cells and alveolar macrophages (21). In this field, the organoid technology has been used to model hereditary lung diseases such as cystic fibrosis with demonstration of gene correction potential (22).

It therefore appears that the unique pluripotent nature of iPSC is a major asset for the generation of organoids-in-a dish toward any types of structures with advantages but also disadvantages as compared to adult-tissue-derived organoids, as summarized in Table 1.

Finally, this highly sophisticated technology with several steps of culture may hold promise not only for the study of infectious diseases (such as SARS-Co-V2) but also for therapeutic purposes as an ATMP product (see below).

\section{ORGANOIDS AS ATMPS: HYPE OR HOPE?}

Given the differentiation potential of iPSC toward almost any organoids, the next question is their potential use as ATMPs. Large scale cGMP grade production of organoids could also lead to the possibility of manufacturing "transplantable" organoids and tissues in the future. From this regard, cardiac and liver tissues could be candidates be generated for transplantation 


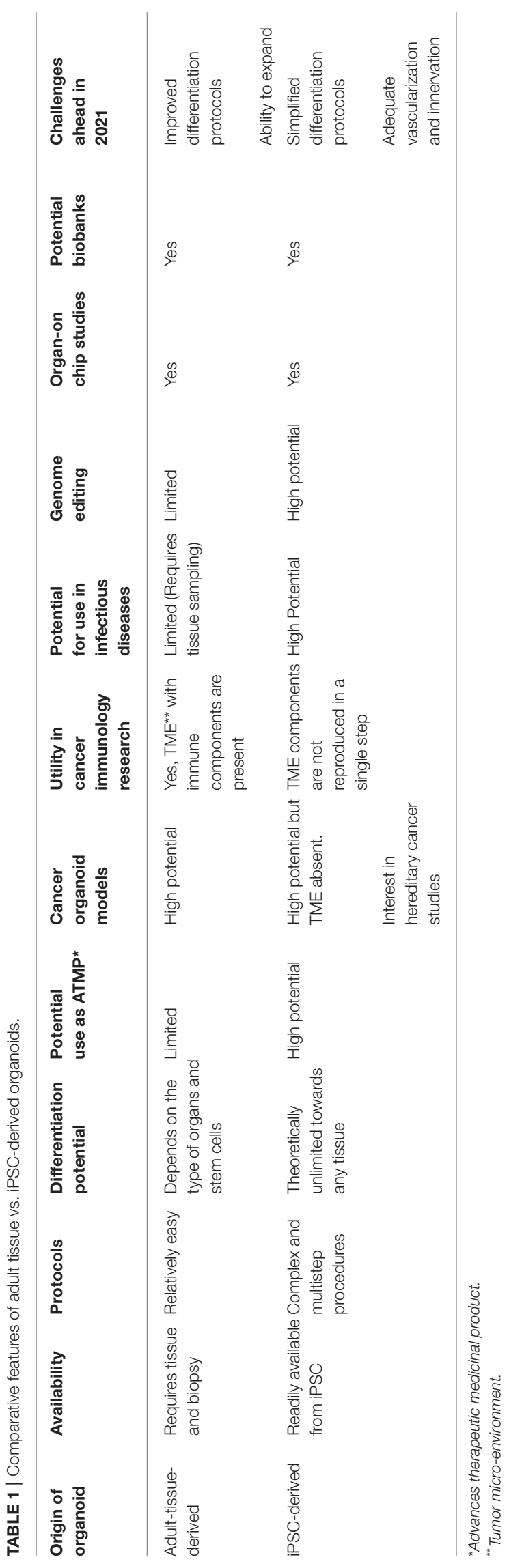

purposes. However, in this field, many efforts are underway and many obstacles remain to be solved. If the use of IPSC-derived corneal cell transplantation has already began in a trial in Japan (23), iPSC-derived organ transplantation in humans is currently a long way from clinical applications but there are studies showing the functional cells can be manufactured. Indeed, in experimental conditions, it has been shown that iPSC-derived kidney nephron structures improve acute renal failure in mice (24). Similarly, erythropoietin-(EPO)-producing iPSC)-derived nephrons could improve anemia associated with terminal kidney failure (25).

In lung diseases such as idiopathic pulmonary fibrosis where the only cure is the lung transplantation, the possibility of generating and transplanting iPSC-derived autologous alveolar epithelial cells could have a significant impact on the prognosis (26).

In the field of diabetes, the transplantation of iPSC-derived islet organoids could be of therapeutic interest in the future as this has been validated in experimental setting (27).

What are the challenges lying ahead before the clinical applications? The vascular organization of future organoids is a major challenge but from patient-specific IPSC, it could be possible to generate after imprinting a brain or heart organoid containing microvasculature derived from HUVEC cells (28).

Similarly, it is necessary to provide in the organoids of the future an adequate innervation system. The possibility of generating iPSC-derived intestinal tissues with an enteric nervous system has been described, generated by combing human intestinal organoids and pluripotent-stem cell derived neural crest cells (29). A combined IPSC-derived hierarchized organoids called "assembloids" have also been described in the hepatobiliary system, generated by inducing the fusion of anterior and posterior gut spheroids, leading a hepato-biliary pancreatic organoids (30). Transplantation of these structures into immunodeficient mice failed however, to give rise to a multi-organ differentiation (30).

\section{IPSC-DERIVED ORGANOIDS IN CANCER}

In the field of cancer, the possibility reproducing cancer of a given organ could be of substantial interest, especially to develop drug screening and for precision medicine. From this regard, cancer organoids could allow to capture genetic heterogeneity as well as the progression-related modifications in a given cancer. Using adult-tissue derived organoids, several studies have shown that organoids generated from the initial tumor as well as from their metastatic counterparts match closely with the original tumor in breast cancer (31). Established from the initial tumor biopsies before any therapy, these organoids arising in vitro within 1-3 months could serve as an in vitro drug screening tools (32). One major drawback of this technology is the fact that it requires obviously the availability of a tissue sample which is not always possible. The growth of the structure is also limited as the tumor biopsy does not always recapitulate the hierarchical subtypes of a tumor. On the other hand, the major advantage of this approach as compared to IPSC-derived modeling is the 
possibility to capture the cellular components of the tumor microenvironment, including immune competent and immunesuppressive cells which could allow the potential responses to immune therapies such as check-point inhibitor therapies (see Table 1).

Although more challenging as compared to adult tissuederived cancer derived organoids, iPSC-generated cancer organoids can be of interest in the study of patients with hereditary cancers. iPSC technology allows also the de novo generation of cancer organoids using genome editing (Figure 2). In the unique situation of the context of hereditary cancers, especially in the carriers of the oncogenic mutation with no established cancer, it may be possible to generate iPSC and to use this oncogene-bearing cell line as an organoid specific of the target tissue such as breast cancer or kidney cancer. This approach was first reported by the group of I. Lemischka using IPSC derived from patents with Li-Fraumeni disease (33).

In our group, we have generated c-MET-mutated iPSC from a patient with hereditary papillary renal cell carcinoma (34). We have shown that kidney organoids generated in vitro recapitulate the transcriptomic features of the primary PRCC of a large cohort of patients. The target genes that we have identified have been also confirmed in the kidney biopsies of patients with PRCC (34). The presence of a given oncogenic mutation in an iPSC line, allows the evaluation of the phenotype generated upon differentiation toward a given pathway and model therefore cancers occurring in several different tissue lineages. We have thus asked whether c-MET mutated iPSC could allow modeling glioblastoma, a tumor in which an overexpression of c-MET has been described in 10\% of cases. We have showed that neural structures derived from these iPSC exhibit transcriptomic features close to that observed in human GBM (35).

The iPSC technology offers also the possibility of generating organoids after induction of specific genomic modifications using molecular manipulation of iPSC (Figure 2). An oncogenic mutation which can be induced in the pluripotent state, can then be propagated with generation of a "transformed organoid" as this has been shown for modeling glioblastoma (36). More recently, it has been shown that overexpression of KRAS G21D oncogene in pancreas acinar cells allow development of pancreas cancer in vitro (37) Similarly, recent work showed the possibility of generating in iPSC-derived RAS-mutated alveolar type 2 (AT2) cells, the induction of a genomic pathways similar to lung adenocarcinoma which is a driver gene in $30 \%$ cases (38). This study allowed to determine the early genomic changes occurring in the AT2 cells some of which were similar to transcriptomic features of in primary lung adenocarcinoma such as overexpression of Sox9 (38). Thus, iPSC-derived cancer organoid technology is expected to expand during the next decade, with several models developed for other cancers (39, 40) with increasing implication of microfluidic technology to study drug sensitivity (41). These technologies will also benefit from the molecular analyses and bioinformatics techniques with discovery of novel targets leading to therapeutic intervention $(34,42)$.

\section{USE OF IPSC-DERIVED ORGANOIDS IN INFECTIOUS DISEASES}

Organoids appear today as major experimental tools for determining target cells and pathophysiology of viral infections. This concept has been successfully applied to Zika virus (43-45) and more recently to SARS-Cov-2 infections. In fact, in a very short period of time, the availability of these bioengineering tools led to their exponential use for several research teams worldwide to identify target cells for COVID-19 entry, and to evaluate the potential therapeutics, and vaccine approaches.

Using a large number of organoids derived from iPSC, it has been shown that the organoids such as pancreas are highly permissive to the virus (46).

Tiwari et al. have generated iPSC-derived brain and lung organoids to study the virus entry into different cell populations and their differential responses to COVID-19 infection (47) In particular they have shown that as compared to lung organoid derived cells, neural progenitors and astrocytes expressing low levels of ACE2 were not permissive to COVID-19, a finding that has been documented in pluripotent stem cells (47). The iPSCorganoid technology represents also a major tool to study drugs that best allow inhibition of virus entry into cells of different origin (48). Human IPSC-derived organoid technology allows also, by the combined use of CRUSPR CAS9 techniques, to determine the genetic susceptibility of human populations to Covid_19 (49). Indeed a unique sNP found in the $3^{\prime}$ UTR of the Furin gene has been shown to influence the infection of lung and neuronal cells by Covid 19. These technologies could therefore help to define populations which could be an increased risk for Covid 19 infection (49). One other interesting potential use of organoid technology in the field of infectious diseases is the use of gastric organoids to study the infection by Helicobacter Pylori $(50,51)$.

\section{PERSPECTIVES AND CHALLENGES AHEAD}

Organoid technology, developed initially from the primary normal or diseased tissues/organs has achieved a novel major perspective by the use of iPSC technology, which offers the possibility of genomic editing and theoretically an unlimited proliferation and differentiation potential. As summarized, the technology holds tremendous potential but several hurdles remain, explaining its current limitations. For larger medical applications, better differentiation protocols are needed. The fact that cells organize themselves in 3D conditions suggest that some cell to cell interactions must occur to lead to pre-organoid structures called 'aggregates" and to the phenomenon of symmetry breaking which occurs during normal embryonic development.

One other limitation of iPSC-derived organoids is the fact that they do not represent the typical environments which are usually found in tissues, especially in cancer in which a particular immune-suppressive microenvironment is present. Similarly, the application of this organoid technology in the future kidney 

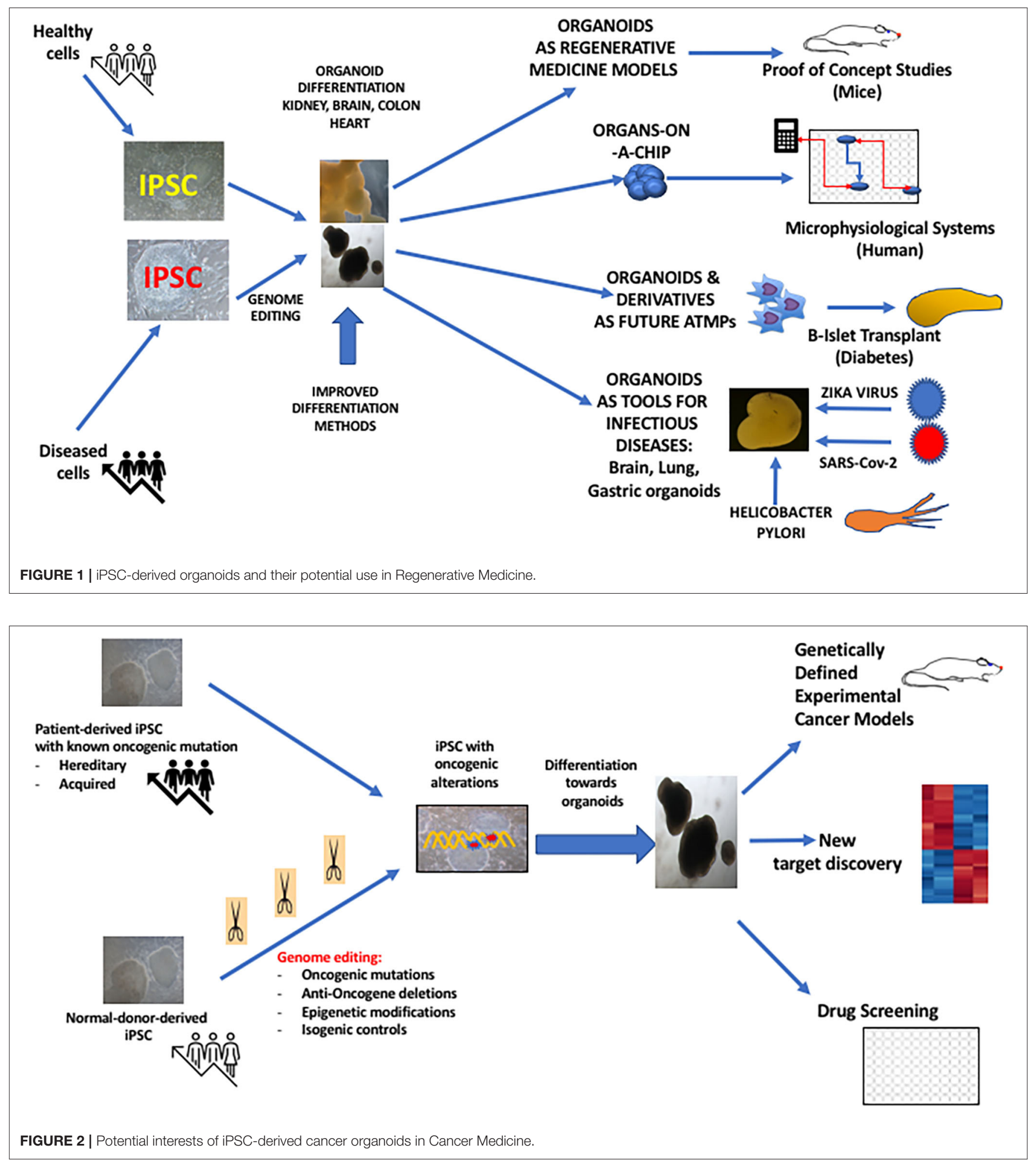

transplants, will require the possibility of generating a functional vasculature but also a urine drainage system, which is not yet been achieved.

Thus, the generation a functional vasculature within the in vitro generated organoids is a significant challenge. Similary, organoids might miss some changes related to aging of the organ especially when generated from iPSC. A recently described human Organoid Atlas could be of major help to generate a "catalog" for human organoids including the standardization of their methodology. Single cell transcriptome analyses as well as spatial profiling will be of major areas of research during the next decade. 
A H2020 project is currently in progress project within the human cell atlas project (https://hca-organoid.eu).

Finally some ethical issues might need to be discussed in the future with regard to the generation and the use of reprogrammed cells but also with regard to the creation of complex and increasingly sophisticated iPSC-derived human organoids (52). Indeed, such ethical issues will need to be discussed for example, with the possibility of generating complex brain organoids, complex integrated systems or early developmental structures such as amniotic sacs (53). These complex integrated systems have already been developed using microphysiological systems allowing organoids to be used for emulation of human biology in "human-organs-on-chips" systems and will pave the way for the drastic reduction of animal use for drug discovery experiments $(54,55)$.

\section{REFERENCES}

1. Clevers H. Modeling development and disease with organoids. Cell. (2016) 165:1586-97. doi: 10.1016/j.cell.2016.05.082

2. Takahashi K, Yamanaka S. Induction of pluripotent stem cells from mouse embryonic and adult fibroblast cultures by defined factors. Cell. (2006) 126:663-76. doi: 10.1016/j.cell.2006.07.024

3. Takahashi K, Tanabe K, Ohnuki M, Narita M, Ichisaka T, Tomoda K, et al. Induction of pluripotent stem cells from adult human fibroblasts by defined factors. Cell. (2007) 131:861-72. doi: 10.1016/j.cell.2007.11.019

4. Karagiannis P, Takahashi K, Saito M, Yoshida Y, Okita K, Watanabe A, et al. Induced pluripotent stem cells and their use in human models of disease and development. Physiol Rev. (2019) 99:79-114. doi: 10.1152/physrev.00039.2017

5. Sato T, Vries RG, Snippert HJ, van de Wetering M, Barker N, Stange DE, et al. Single Lgr5 stem cells build crypt-villus structures in vitro without a mesenchymal niche. Nature. (2009) 459:262-5. doi: 10.1038/nature07935

6. Garreta E, Kamm RD, Chuva de Sousa Lopes SM, Lancaster MA, Weiss R, Trepat X, et al. Rethinking organoid technology through bioengineering. Nat Mater. (2021) 20:145-55. doi: 10.1038/s41563-020-00804-4

7. Spence JR, Mayhew CN, Rankin SA, Kuhar MF, Vallance JE, Tolle K, et al. Directed differentiation of human pluripotent stem cells into intestinal tissue in vitro. Nature. (2011) 470:105-9. doi: 10.1038/nature09691

8. Nakano T, Ando S, Takata N, Kawada M, Muguruma K, Sekiguchi K, et al. Self-formation of optic cups and storable stratified neural retina from human ESCs. Cell Stem Cell. (2012) 10:771-85. doi: 10.1016/j.stem.2012.05.009

9. Lancaster MA, Renner M, Martin CA, Wenzel D, Bicknell LS, Hurles ME, et al. Cerebral organoids model human brain development and microcephaly. Nature. (2013) 501:373-9. doi: 10.1038/nature12517

10. Giandomenico SL, Sutcliffe M, Lancaster MA. Generation and long-term culture of advanced cerebral organoids for studying later stages of neural development. Nat Protoc. (2021) 16:579602. doi: 10.1038/s41596-020-00433-w

11. Renner M, Lancaster MA, Bian S, Choi H, Ku T, Peer A, et al. Self-organized developmental patterning and differentiation in cerebral organoids. EMBO J. (2017) 36:1316-29. doi: 10.15252/embj.201694700

12. Birey F, Andersen J, Makinson CD, Islam S, Wei W, Huber N, et al. Assembly of functionally integrated human forebrain spheroids. Nature. (2017) 545:549. doi: 10.1038/nature22330

13. Takasato M, Er PX, Chiu HS, Maier B, Baillie GJ, Ferguson C, et al. Kidney organoids from human iPS cells contain multiple lineages and model human nephrogenesis. Nature. (2015) 526:564-8. doi: 10.1038/nature15695

14. Kim JW, Nam SA, Seo E, Lee JY, Kim D, Ju JH, et al. Human kidney organoids model the tacrolimus nephrotoxicity and elucidate the role of autophagy. Korean J Intern Med. (2020) 1-17. doi: 10.3904/kjim.2020.323

15. Takebe T, Sekine K, Enomura M, Koike H, Kimura M, Ogaeri T, et al. Vascularized and functional human liver from an iPSC-derived organ bud transplant. Nature. (2013) 499:481-4. doi: 10.1038/nature12271
Overall, both adult tissue and iPSC-derived organoids offer an unprecedented complementary information in almost all areas of medicine with accelerated discovery of novel targets and potentially as a therapeutic ATMP modality in the future.

\section{AUTHOR CONTRIBUTIONS}

All authors listed have made a substantial, direct and intellectual contribution to the work, and approved it for publication.

\section{FUNDING}

This study was performed by the internal funds of INGESTEM.

16. Hannan NR, Segeritz CP, Touboul T, Vallier, L. Production of hepatocyte like cells from human pluripotent stem cells. Nat. Protocols. (2013) 8:43037. doi: 10.1038/nprot.2012.153

17. Nantasanti S, de Bruin A, Rothuizen J, Penning LC, Schotanus BA. Concise review: organoids are a powerful tool for the study of liver disease and personalized treatment design in humans and animals. Stem Cells Transl Med. (2016) 5:325-30. doi: 10.5966/sctm.2015-0152

18. Cyganek L, Tiburcy M, Sekeres K, Gerstenberg K, Bohnenberger H, Lenz C, et al. Deep phenotyping of human induced pluripotent stem cell-derived atrial and ventricular cardiomyocytes. JCI Insight. (2018) 3:e99941. doi: 10.1172/jci.insight.99941

19. Nugraha B, Buono MF, von Boehmer L, Hoerstrup SP, Emmert MY. Human cardiac organoids for disease modeling. Clin Pharmacol Ther. (2019) 105:7985. doi: $10.1002 /$ cpt.1286

20. Basil MC, Morrisey EE. Lung regeneration: a tale of mice and men. Semin Cell Dev Biol. (2020) 100:88-100. doi: 10.1016/j.semcdb.2019.11.006

21. Dye BR, Hill DR, Ferguson MA, Tsai YH, Nagy MS, Dyal R, et al. In vitro generation of human pluripotent stem cell derived lung organoids. Elife. (2015) 4:e05098. doi: 10.7554/eLife.05098

22. Firth AL, Menon T, Parker GS, Qualls SJ, Lewis BM, Ke E, et al. Functional gene correction for cystic fibrosis in lung epithelial cells generated from patient iPSCs. Cell Rep. (2015) 12:1385-90. doi: 10.1016/j.celrep.2015. 07.062

23. Hayashi R, Ishikawa Y, Sasamoto Y, Katori R, Nomura N, Ichikawa $\mathrm{T}$, et al. Co-ordinated ocular development from human iPS cells and recovery of corneal function. Nature. (2016) 531:376-80. doi: 10.1038/nature 17000

24. Toyohara T, Mae S, Sueta S, Inoue T, Yamagishi Y, Kawamoto T, et al. Cell therapy using human induced pluripotent stem cell-derived renal progenitors ameliorates acute kidney injury in mice. Stem Cells Transl Med. (2015) 4:980-92. doi: 10.5966/sctm.2014-0219

25. Osafune K. iPSC technology-based regenerative medicine for kidney diseases. Clin Exp Nephrol. (2021) 25:574-84. doi: 10.1007/s10157-021-02030-x

26. Oglesby IK, Schweikert A, Fox B, Redmond C, Donnelly SC, Hurley K. Lung organoids and other preclinical models of pulmonary fibrosis. QJM. (2021) 114:167-73. doi: 10.1093/qjmed/hcaa281

27. Yoshihara E, O'Connor C, Gasser E, Wei Z, Oh TG, Tseng TW, et al. Immune-evasive human islet-like organoids ameliorate diabetes. Nature. (2020) 586:606-11. doi: 10.1038/s41586-020-2631-z

28. Skylar-Scott MA, Uzel SGM, Nam LL, Ahrens JH, Truby RL, Damaraju S, et al. Biomanufacturing of organ-specific tissues with high cellular density and embedded vascular channels. Sci Adv. (2019) 5:eaaw2459. doi: 10.1126/sciadv.aaw2459

29. Workman MJ, Mahe MM, Trisno S, Poling HM, Watson CL, Sundaram $\mathrm{N}$, et al. Engineered human pluripotent-stem-cell-derived intestinal tissues with a functional enteric nervous system. Nat Med. (2017) 23:4959. doi: $10.1038 / \mathrm{nm} .4233$ 
30. Koike H, Iwasawa K, Ouchi R, Maezawa M, Giesbrecht K, Saiki N, et al. Modelling human hepato-biliary-pancreatic organogenesis from the foregutmidgut boundary. Nature. (2019) 574:112-6. doi: 10.1038/s41586-019-1598-0

31. Sachs N, de Ligt J, Kopper O, Gogola E, Bounova G, Weeber F, et al. A living biobank of breast cancer organoids captures disease heterogeneity. Cell. (2018) 172:373-86.e10. doi: 10.1016/j.cell.2017.11.010

32. Driehuis E, Kretzschmar K, Clevers H. Establishment of patient-derived cancer organoids for drug-screening applications. Nat Protoc. (2020) 15:3380409. doi: 10.1038/s41596-020-0379-4

33. Lee DF, Su J, Kim HS, Chang B, Papatsenko D, Zhao R, et al. Modeling familial cancer with induced pluripotent stem cells. Cell. (2015) 161:24054. doi: 10.1016/j.cell.2015.02.045

34. Hwang JW, Desterke C, Féraud O, Richard S, Ferlicot S, Verkarre $\mathrm{V}$, et al. iPSC-derived embryoid bodies as models of c-Met-mutated hereditary papillary renal cell carcinoma. Int J Mol Sci. (2019) 20:4867. doi: 10.3390/ijms20194867

35. Hwang JW, Loisel-Duwattez J, Desterke C, Latsis T, Pagliaro S, Griscelli F, et al. A novel neuronal organoid model mimicking glioblastoma (GBM) features from induced pluripotent stem cells (iPSC). Biochim Biophys Acta Gen Subj. (2020) 1864:129540. doi: 10.1016/j.bbagen.2020.129540

36. Sancho-Martinez I, Nivet E, Xia Y, Hishida T, Aguirre A, Ocampo A, et al. Establishment of human iPSC-based models for the study and targeting of glioma initiating cells. Nat Commun. (2016) 7:10743. doi: $10.1038 /$ ncomms 10743

37. Huang L, Desai R, Conrad DN, Leite NC, Akshinthala D, Lim CM, et al. Commitment and oncogene-induced plasticity of human stem cell-derived pancreatic acinar and ductal organoids. Cell Stem Cell. (2021) 28:1090104.e6. doi: 10.1016/j.stem.2021.03.022

38. Dost AFM, Moye AL, Vedaie M, Tran LM, Fung E, Heinze D, et al. Organoids model transcriptional hallmarks of oncogenic KRAS activation in lung epithelial progenitor cells. Cell Stem Cell. (2020) 27:66378.e8. doi: 10.1016/j.stem.2020.07.022

39. Hepburn AC, Sims CHC, Buskin A, Heer R. Engineering prostate cancer from induced pluripotent stem cells-new opportunities to develop preclinical tools in prostate and prostate cancer studies. Int J Mol Sci. (2020) 21:905. doi: 10.3390/ijms21030905

40. Crespo M, Vilar E, Tsai SY, Chang K, Amin S, Srinivasan T, et al. Colonic organoids derived from human induced pluripotent stem cells for modeling colorectal cancer and drug testing. Nat Med. (2017) 23:87884. doi: $10.1038 / \mathrm{nm} .4355$

41. Shirure VS, Bi Y, Curtis MB, Lezia A, Goedegebuure MM, Goedegebuure SP, et al. Tumor-on-a-chip platform to investigate progression and drug sensitivity in cell lines and patient-derived organoids. Lab Chip. (2018) 18:3687-702. doi: 10.1039/C8LC00596F

42. Kim M, Mun H, Sung CO, Cho EJ, Jeon HJ, Chun SM, et al. Patient-derived lung cancer organoids as in vitro cancer models for therapeutic screening. Nat Commun. (2019) 10:3991. doi: 10.1038/s41467-019-11867-6

43. Lanko K, Eggermont K, Patel A, Kaptein S, Delang L, Verfaillie CM, et al. Replication of the Zika virus in different iPSC-derived neuronal cells and implications to assess efficacy of antivirals. Antiviral Res. (2017) 145:8286. doi: 10.1016/j.antiviral.2017.07.010

44. Ledur PF, Karmirian K, Pedrosa CDSG, Souza LRQ, Assis-de-Lemos G, Martins TM, et al. Zika virus infection leads to mitochondrial failure, oxidative stress and DNA damage in human iPSC-derived astrocytes. Sci Rep. (2020) 10:1218. doi: 10.1038/s41598-020-57914-X

45. Zhou T, Tan L, Cederquist GY, Fan Y, Hartley BJ, Mukherjee S, et al. Highcontent screening in hPSC-neural progenitors identifies drug candidates that inhibit Zika virus infection in fetal-like organoids and adult brain. Cell Stem Cell. (2017) 21:274-83.e5. doi: 10.1016/j.stem.2017.06.017

46. Yang L, Han Y, Nilsson-Payant BE, Gupta V, Wang P, Duan X, et al. A human pluripotent stem cell-based platform to study SARS-CoV-2 tropism and model virus infection in human cells and organoids. Cell Stem Cell. (2020) 27:125-36.e7. doi: 10.1016/j.stem.2020.06.015

47. Tiwari SK, Wang S, Smith D, Carlin AF, Rana TM. Revealing tissuespecific SARS-CoV-2 infection and host responses using human stem cellderived lung and cerebral organoids. Stem Cell Reports. (2021) 16:43745. doi: 10.1016/j.stemcr.2021.02.005

48. Desterke C, Griscelli F, Imeri J, Marcoux P, Lemonnier T, Latsis T, et al. Molecular investigation of adequate sources of mesenchymal stem cells for cell therapy of COVID-19-associated organ failure. Stem Cells Transl Med. (2021) 10:568-71. doi: 10.1002/sctm.20-0189

49. Dobrindt K, Hoagland DA, Seah C, Kassim B, O’Shea CP, Murphy $\mathrm{A}$, et al. Common genetic variation in humans impacts in vitro susceptibility to SARS-CoV-2 infection. Stem Cell Rep. (2021) 16:505-18. doi: 10.1016/j.stemcr.2021.02.010

50. McCracken KW, Catá EM, Crawford CM, Sinagoga KL, Schumacher $\mathrm{M}$, Rockich BE, et al. Modelling human development and disease in pluripotent stem-cell-derived gastric organoids. Nature. (2014) 516:4004. doi: 10.1038/nature13863

51. Engevik KA, Matthis AL, Montrose MH, Aihara E. Organoids as a model to study infectious disease. Methods Mol Biol. (2018) 1734:7181. doi: 10.1007/978-1-4939-7604-1_8

52. Hyun, I. Engineering ethics and self-organizing models of human development: opportunities and challenges. Cell Stem Cell. (2017) 21:718-20. doi: 10.1016/j.stem.2017.09.002

53. Shao Y, Taniguchi K, Townshend RF, Miki T, Gumucio DL, Fu J. A pluripotent stem cell-based model for post-implantation human amniotic sac development. Nat Commun. (2017) 8:208. doi: 10.1038/s41467-017-00236-w

54. Marx U, Andersson TB, Bahinski A, Beilmann M, Beken S, Cassee FR, et al. Biology-inspired microphysiological system approaches to solve the prediction dilemma of substance testing. ALTEX. (2016) 33:272321. doi: 10.14573/altex.1603161

55. Xiao S, Coppeta JR, Rogers HB, Isenberg BC, Zhu J, Olalekan SA, et al. A microfluidic culture model of the human reproductive tract and 28-day menstrual cycle. Nat Commun. (2017) 8:14584. doi: 10.1038/ncomms14584

Conflict of Interest: The authors declare that the research was conducted in the absence of any commercial or financial relationships that could be construed as a potential conflict of interest.

Publisher's Note: All claims expressed in this article are solely those of the authors and do not necessarily represent those of their affiliated organizations, or those of the publisher, the editors and the reviewers. Any product that may be evaluated in this article, or claim that may be made by its manufacturer, is not guaranteed or endorsed by the publisher.

Copyright (c) 2021 Turhan, Hwang, Chaker, Tasteyre, Latsis, Griscelli, Desterke and Bennaceur-Griscelli. This is an open-access article distributed under the terms of the Creative Commons Attribution License (CC BY). The use, distribution or reproduction in other forums is permitted, provided the original author(s) and the copyright owner(s) are credited and that the original publication in this journal is cited, in accordance with accepted academic practice. No use, distribution or reproduction is permitted which does not comply with these terms. 